\title{
Bulk Mediated Surface Diffusion: Finite System Case
}

\author{
Jorge A. Revelli, ${ }^{1}$ Carlos. E. Budde, ${ }^{2}$ Domingo Prato ${ }^{2}$ and Horacio S. Wio ${ }^{1,3}$ \\ ${ }^{1}$ Grupo de Física Estadística, Centro Atómico Bariloche and Instituto Balseiro, \\ 8400 San Carlos de Bariloche, Argentina \\ ${ }^{2}$ Facultad de Matemáticas, Astronomía y Física, Universidad Nacional de Córdoba \\ 5000 Córdoba, Argentina \\ ${ }^{3}$ Departament de Física, Universitat de les Illes Balears and IMEDEA \\ E-07122 Palma de Mallorca, Spain
}

\begin{abstract}
We address the dynamics of adsorbed molecules (a fundamental issue in surface physics) within the framework of a Master Equation scheme, and study the diffusion of particles in a finite cubic lattice whose boundaries are at the $z=1$ and the $z=L$ planes where $L=2,3,4, \ldots$, while the $x$ and $y$ directions are unbounded. As we are interested in the effective diffusion process at the interface $z=1$, we calculate analytically the conditional probability for finding the system on the $z=1$ plane as well as the surface dispersion as a function of time and compare these results with Monte Carlo simulations finding an excellent agreement.
\end{abstract}

PACS numbers: 05.40.Fb, 02.50.Ey, 05.10.Ln, 46.65.+g 


\section{INTRODUCTION}

The dynamics of adsorbed molecules is a fundamental issue in interface science [1, 2, 3, 4, 5, 6, 7, 8, 9, 10] and is also crucial in a large number of emerging technologies [3, 11]. Adsorption at the solid-liquid interfaces arises, for instance, in many biological contexts involving protein deposition [12, 13, 14], in solutions or melts of synthetic macromolecules [15, 16, 17, 18], in colloidal dispersions [19], and in the manufacture of self-assembly monoand multi-layers [11, 20, 21, 22].

Experimental studies of surfactant molecules [5, 6, 7], proteins [9, 10, 23] and synthetic polymers [8, 10] confined to interfaces have identified different types of surface translational motions. One is in-surface self-diffusion of individual molecules, which has been investigated with fluorescence recovery after photobleaching (FRAP) methods [4, 10, 23]. Measured selfdiffusivity at liquid-solid interfaces are much smaller than bulk values [23] and similar to bulk values in liquid-gas cases [10]. A second source of motion, exclusive to the liquid-fluid interface, is surface visco-elasticity. Adsorbed surface phases possess compressibility and viscosities which govern the dynamics of the surface density waves. Their relaxation kinetics have been intensively studied experimentally [5]-7] and theoretically 1, 2, 24, 25], with direct viscoelastic measurement [5] and surface light scattering studies [4, 24, 25] providing considerable support for current theories.

In this paper we explore another mechanism called bulk-mediated surface diffusion. This mechanism arises at interfaces separating a liquid bulk phase and a second phase which may be either solid, liquid, or gaseous. Whenever the adsorbed species are soluble in the liquid bulk, adsorption-desorption processes occur continuously. These processes generate surface displacement because desorbed molecules undergo Fickian diffusion in the liquid's bulk, and are later re-adsorbed elsewhere. When this process is repeated many times, an effective diffusion results for the molecules on the surface. The importance of bulk-surface exchange in relaxing homogeneous surface density perturbations is experimentally well established [1, 2, 5, 9, 26].

Here, unlike our previous work [27] on the adsorption-desorption mechanism, we assume that the bulk is finite. The main goal of this work is the observation of an effective diffusion process at the interface $z=1$. We calculate analytically its variance, $\left\langle r^{2}(t)\right\rangle$, as a function of time and $P(x, y, z=1 ; t \mid 0,0,1, t=0)$, the conditional probability of being on the surface 
at time $t$ since the particle arrived at $t=0$, that we indicate as $P_{z=1}(t)$.

The organization of the paper is as follows. In the next section we describe the model by means of a set of Master Equations and solve this system in Fourier-Laplace space. After that, we present a special case (the bilayer case) where we can obtain explicit analytical results for $P_{z=1}(t)$ and $\left\langle r^{2}(t)\right\rangle$. Next we compare these theoretical results with numeric Monte Carlo simulations. Moreover, we present results obtained by numerical inverse transformation of the equations for $\left\langle r^{2}\right\rangle$ and $P_{z=1}$ for the cases with more layers. In the last section we discuss the results and draw some conclusions.

\section{THE MODEL}

Let us start with the problem of a particle making a random walk in a finite cubic lattice. The bulk is bounded in the $z$ direction where the particles can move from $z=1$ to $z=L$. The $x$ and $y$ directions remain unbounded. The position of the walker is defined by a vector $\vec{r}$ whose components are denoted by the integer numbers $n, m, l$ corresponding to the directions $x, y$ and $z$ respectively.

The probability that the walker is at $(n, m, l)$ for time $t$ given it was at $\left(0,0, l_{0}\right)$ at $t=0$, $P\left(n, m, l ; t \mid 0,0, l_{0}, t=0\right)=P(n, m, l ; t)$, satisfies the following master equation

$$
\begin{aligned}
\dot{P}(n, m, l ; t)= & \gamma P(n, m, l+1 ; t)-\delta P(n, m, l ; t) \\
& +\alpha^{1}[P(n-1, m, l ; t)+P(n+1, m, l ; t)-2 P(n, m, l ; t)] \\
& +\beta^{1}[P(n, m-1, l ; t)+P(n, m+1, l ; t)-2 P(n, m, l ; t)], \quad \text { for } l=1 \\
\dot{P}(n, m, l ; t)= & \alpha[P(n-1, m, l ; t)+P(n+1, m, l ; t)-2 P(n, m, l ; t)] \\
& +\beta[P(n, m-1, l ; t)+P(n, m+1, l ; t)-2 P(n, m, l ; t)] \\
& +\gamma P(n, m, l+1 ; t)+\delta P(n, m, l-1 ; t)-2 \gamma P(n, m, l ; t), \quad \text { for } l=2 \\
\dot{P}(n, m, l ; t)= & \alpha[P(n-1, m, l ; t)+P(n+1, m, l ; t)-2 P(n, m, l ; t)] \\
& +\beta[P(n, m-1, l ; t)+P(n, m+1, l ; t)-2 P(n, m, l ; t)] \\
& +\gamma[P(n, m, l+1 ; t)+P(n, m, l-1 ; t)-2 \gamma[P(n, m, l ; t)], \quad \text { for } 3 \leq l \leq L-1 \\
\dot{P}(n, m, l ; t)= & \gamma P(n, m, l-1 ; t)-\gamma P(n, m, l ; t) \quad \\
& +\alpha[P(n-1, m, l ; t)+P(n+1, m, l ; t)-2 P(n, m, l ; t)] \quad \\
& +\beta[P(n, m-1, l ; t)+P(n, m+1, l ; t)-2 P(n, m, l ; t)], \quad \text { for } l=L .
\end{aligned}
$$


where $\alpha, \beta$ and $\gamma$ are the bulk transition probabilities per unit time in the $x, y$ and $z$ directions respectively, and $\delta$ is the desorption probability per unit time from the boundary plane $z=1$.

It is important to note that the model presented in Eq. (11), allows the possibility that the particles can move in the plane $z=1$ with temporal frequencies $\alpha^{1}$ in the $x$ direction and $\beta^{1}$ in the $y$ direction. If these temporal frequencies are equal to zero, the motion through the $z=1$ plane is exclusively due to the dynamics across the bulk. In addition we can observe that this is a finite set of $L$ equations. This fact establishes an important difference with the infinite set of equations presented in [27], a crucial difference because this generates different solutions to the problem. In order to solve this finite set of equations, we take the Fourier transform with respect to the $x$ and $y$ variables, and the Laplace transform in the $t$ variable. After these transformations, we obtain the following set of equations

$$
\begin{aligned}
s G\left(k_{x}, k_{y}, l ; s\right)-P\left(k_{x}, k_{y}, l, t=0\right)= & \gamma G\left(k_{x}, k_{y}, l+1 ; s\right) \\
& -\delta G\left(k_{x}, k_{y}, l ; s\right)+A^{1}\left(k_{x}, k_{y}\right) G\left(k_{x}, k_{y}, l ; s\right), \quad \text { for } l=1 \\
s G\left(k_{x}, k_{y}, l ; s\right)-P\left(k_{x}, k_{y}, l, t=0\right)= & A\left(k_{x}, k_{y}\right) G\left(k_{x}, k_{y}, l ; s\right)+\delta G\left(k_{x}, k_{y}, l-1 ; s\right) \\
& +\gamma G\left(k_{x}, k_{y}, l+1 ; s\right)-2 \gamma G\left(k_{x}, k_{y}, l ; s\right), \quad \text { for } l=2 \\
s G\left(k_{x}, k_{y}, l ; s\right)-P\left(k_{x}, k_{y}, l, t=0\right)= & A\left(k_{x}, k_{y}\right) G\left(k_{x}, k_{y}, l ; s\right)+\gamma\left[G\left(k_{x}, k_{y}, l-1 ; s\right)\right. \\
& \left.+\gamma G\left(k_{x}, k_{y}, l+1 ; s\right)-2 \gamma G\left(k_{x}, k_{y}, l ; s\right)\right], \quad \text { for } 3 \leq l \leq L-1 \\
s G\left(k_{x}, k_{y}, l ; s\right)-P\left(k_{x}, k_{y}, l, t=0\right)= & A\left(k_{x}, k_{y}\right) G\left(k_{x}, k_{y}, l ; s\right) \quad \\
& -\gamma G\left(k_{x}, k_{y}, l ; s\right)+\gamma G\left(k_{x}, k_{y}, l-1 ; s\right), \quad \text { for } l=L
\end{aligned}
$$

where we have defined

$$
\begin{aligned}
G\left(k_{x}, k_{y}, l ; s\right) & =G\left(k_{x}, k_{y}, l ; s \mid 0,0, l_{0} ; t=0\right) \\
& =\int_{0}^{\infty} e^{-s t} \sum_{n, m,-\infty}^{\infty} e^{k_{x} n+k_{y} m} P(n, m, l ; t) d t \\
& =L\left[\sum_{n, m,-\infty}^{\infty} e^{k_{x} n+k_{y} m} P(n, m, l ; t)\right],
\end{aligned}
$$

$L$ indicates the Laplace transform of the quantity inside the brackets, and

$$
\begin{aligned}
A\left(k_{x}, k_{y}\right) & =2 \alpha\left[\cos \left(k_{x}\right)-1\right]+2 \beta\left[\cos \left(k_{y}\right)-1\right], \\
A^{1}\left(k_{x}, k_{y}\right) & =2 \alpha^{1}\left[\cos \left(k_{x}\right)-1\right]+2 \beta^{1}\left[\cos \left(k_{y}\right)-1\right] .
\end{aligned}
$$


Using the matrix formalism, Eq. (21) can be written as

$$
[s \tilde{I}-\tilde{H}] \tilde{G}=\delta_{l l_{0}}=\tilde{I}_{l l_{0}},
$$

where $\tilde{G}$ is an $L \times L$ array that has the following components

$$
\tilde{G}_{l l_{0}}=\left[G\left[k_{x}, k_{y}, l ; s \mid n, m, l_{0} ; t_{0}\right]\right]
$$

$\tilde{I}$ is the identity matrix and $\tilde{H}$ is a tri-diagonal matrix whose components are

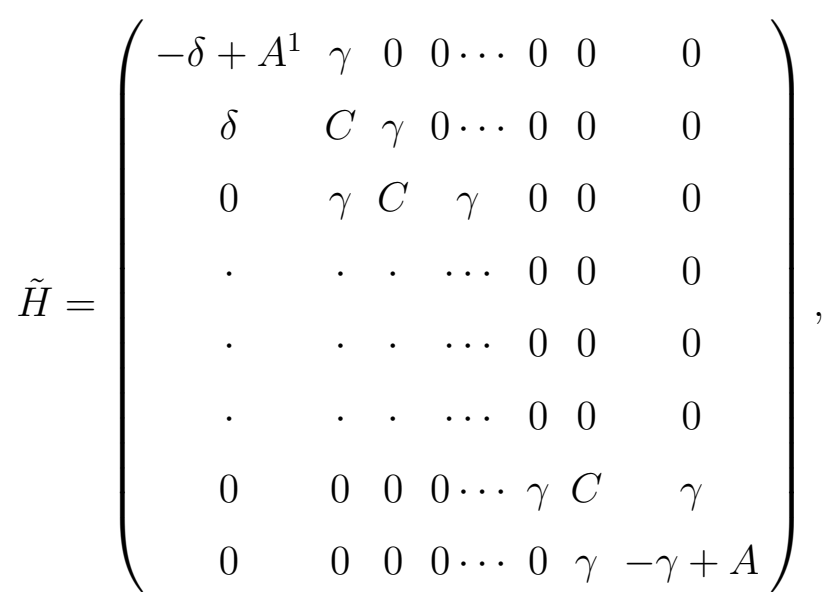

The $C$ parameter is defined as

$$
C=-2 \gamma+A\left(k_{x}, k_{y}\right)
$$

In order to find the solution to the Eq. (6), we decompose the $\tilde{H}$ matrix according to

$$
\tilde{H}=A\left(k_{x}, k_{y}\right) \tilde{I}+\tilde{H}_{0}+\tilde{H}_{1}+\tilde{H}_{2}
$$

where

$$
\begin{gathered}
\tilde{H}_{0}=\left(\begin{array}{ccccccc}
-2 \gamma & \gamma & 0 & 0 \cdots & 0 & 0 & 0 \\
\gamma & -2 \gamma & \gamma & 0 \cdots & 0 & 0 & 0 \\
0 & \gamma & -2 \gamma & \gamma \cdots & 0 & 0 & 0 \\
\cdot & \cdot & \cdot & \cdots & 0 & 0 & 0 \\
\cdot & \cdot & \cdot & \cdots & 0 & 0 & 0 \\
\cdot & \cdot & \cdot & \cdots & 0 & 0 & 0 \\
0 & \cdot & \cdot & \cdots & \gamma & -2 \gamma & \gamma \\
0 & \cdot & \cdot & \cdots & 0 & \gamma & -\gamma
\end{array}\right), \\
\left(\tilde{H}_{1}\right)_{i j}=\Delta_{1}\left\{\begin{array}{l}
1 \text { if } \mathrm{i}=\mathrm{j}=1 \\
0 \text { otherwise }
\end{array}\right.
\end{gathered}
$$




$$
\left(\tilde{H}_{2}\right)_{i j}=\Delta_{2}\left\{\begin{array}{l}
1 \text { if } \mathrm{i}=1 \text { and } \mathrm{j}=2 \\
0 \text { otherwise }
\end{array}\right.
$$

and

$$
\begin{aligned}
& \Delta_{1}=-\delta-\left[-2 \gamma+A\left(k_{x}, k_{y}\right)-A^{1}\left(k_{x}, k_{y}\right)\right], \\
& \Delta_{2}=\delta-\gamma .
\end{aligned}
$$

We also define

$$
\begin{aligned}
\tilde{G}^{0} & =\left[s \tilde{I}-\left(A\left(k_{x}, k_{y}\right) \tilde{I}+\tilde{H}_{0}\right)\right]^{-1}, \\
\tilde{G}^{1} & =\left[s \tilde{I}-\left(A\left(k_{x}, k_{y}\right) \tilde{I}+\tilde{H}_{0}+\tilde{H}_{1}\right)\right]^{-1} .
\end{aligned}
$$

A formal solution of the Eq. (6) is

$$
\tilde{G}=[s \tilde{I}-\tilde{H}]^{-1}
$$

We can show, by applying the Dyson formula 31], that

$$
\begin{gathered}
\tilde{G}_{l l_{0}}=\tilde{G}_{l l_{0}}^{1}+\frac{\Delta_{2} \tilde{G}_{l 2}^{1} \tilde{G}_{1 l_{0}}^{1}}{1-\Delta_{2} \tilde{G}_{12}^{1}}, \\
\tilde{G}_{l l_{0}}^{1}=\tilde{G}_{l l_{0}}^{0}+\frac{\Delta_{1} \tilde{G}_{l 1}^{0} \tilde{G}_{1 l_{0}}^{0}}{1-\Delta_{1} \tilde{G}_{11}^{0}} .
\end{gathered}
$$

The solution for $\tilde{G}_{l l_{0}}^{0}$ can be obtained analytically [28]. The result is

$$
\tilde{G}_{l l_{0}}^{0}=\sum_{i=0}^{L-1} f_{l i} f_{l_{0} i} \frac{1}{2 \gamma+\left(s-A\left(k_{x}, k_{y}\right)\right)-2 \gamma \cos \left(q_{i}\right)},
$$

where

$$
f_{l i}=K \sin \left(l q_{i}\right)
$$

and

$$
q_{i}=\frac{(2 i+1) \pi}{2 L+1}
$$

The constant $K$ is obtained by exploiting the orthonormality relations for the $f_{l i}$ functions given by

$$
\sum_{i=1} f_{i l} f_{i j}=\delta_{l j}
$$


while the completeness relation for the $f_{l i}$ functions is written as

$$
\sum_{i=1} f_{l i} f_{j i}=\delta_{l j}
$$

The corresponding expression for $K$ is

$$
K=\frac{2}{\sqrt{2 L+1}}
$$

We are now able to find the statistical quantities which describe the diffusion problem over the surface. We are interested in the probability of finding a particle in the site $(m, n, l=1)$ at time $t$ given it was in $(0,0, l=1)$ at $t=0$. This quantity is obtained by applying the inverse Laplace transform on the $\tilde{G}_{11}$ matrix element. Another direct measurable experimental magnitude [29, 30] is the variance $\left(\left\langle r^{2}(t)\right\rangle\right)$ of the probability distribution at time $t$ over the plane

$$
\left\langle r^{2}(t)\right\rangle_{\text {plane }}
$$

This quantity measures the particles dispersion over the surface and has a direct relation to the diffusion coefficient. When $P\left(m, n, l=1 ; t \mid 0,0, l_{0}=1 ; t=0\right)$ is known, the variance is calculated in the following manner

$$
\left\langle r^{2}(t)\right\rangle_{\text {plane }}=\sum_{m, n=-\infty}^{\infty} P\left(m, n, l=1 ; t \mid 0,0, l_{0}=1 ; t=0\right)\left(m^{2}+n^{2}\right) .
$$

In order to obtain this expression, we have assumed symmetric properties for the diffusion along the $x$ and $y$ directions, that is $\langle x(t)\rangle=<y(t)\rangle=0$.

Finally the variance in the Laplace space can be obtained as

$$
<r^{2}(s)>_{\text {plane }}=-\left.\left[\frac{\partial^{2}}{\partial k_{x}^{2}}+\frac{\partial^{2}}{\partial k_{y}^{2}}\right]\left[\tilde{G}_{11}\right]\right|_{k_{x}=k_{y}=0} .
$$

\section{THE BILAYER CASE}

So far, we have developed a general theory describing the important problem of the diffusion of a particle system over a surface which is surrounded by a bounded bulk. The expressions we found, are in the Laplace space and the obtention of the inverse transform is usually a non trivial task.

In this section we restrict the problem to finding a particular solution, considering the case of a system of particles moving inside a bilayer, that is the space formed by the surface 
where we investigate the movement of the particles and a second layer. To obtain the $P\left(m, n, l=1 ; t \mid 0,0, l_{0}=1 ; t=0\right)\left(P_{z=1}(t)\right)$, we take the expression in Eq. (12) with $L=2$ and evaluate for $k_{x}=k_{y}=0$. Then we obtain the inverse Laplace transform. The result is

$$
P_{z=1}(t)=\frac{\gamma}{(\gamma+\delta)}+\frac{\delta}{(\gamma+\delta)} \exp [-(\gamma+\delta) t]
$$

This expression establishes that this probability is only a function of the temporal adsorption and desorption rates and does not depend on the temporal rates on the plane. From this equation, we can also obtain the initial condition of the problem by evaluating Eq. (24) at $t=0$, obtaining $P_{z=1}(t=0)=1$.

If we consider the long time limit, the expression has the following behavior

$$
\lim _{t \rightarrow \infty} P_{z=1}(t)=\frac{\gamma}{\gamma+\delta}
$$

This expression shows that, at this limit, the probability of finding the particle in the $z=1$ plane does not decay to zero (as happens in unbounded systems [27]), but it approaches an asymptotic value which is a function of the ratio of the temporal rates.

If the adsorption rate $\gamma$ is large (compared with the desorption rate $\delta$ ), then the probability is large, the particles go back to the plane frequently. On the other hand, if the desorption rate is large, the particles go away from the surface and so the probability decays. These behaviors are shown and quantified by Eq. (24).

In the case of the variance, the result we have obtained can be decomposed as follows

$$
\left\langle r^{2}(t)\right\rangle=\left\langle r^{2}(t)\right\rangle_{\text {plane }}+\left\langle r^{2}(t)\right\rangle_{v o l}
$$

where

$$
\begin{aligned}
\left\langle r^{2}(t)\right\rangle_{\text {plane }}= & \left(\alpha^{1}+\beta^{1}\right)\left(\frac{-2 \gamma \delta}{(\gamma+\delta)^{3}}(\exp [-(\gamma+\delta) t]-1)\right. \\
& \left.+\frac{2 \gamma \delta+\delta^{2}}{(\gamma+\delta)^{2}} t \exp [-(\gamma+\delta) t]+\frac{\gamma^{2}}{(\gamma+\delta)^{2}} t\right) \\
\left\langle r^{2}(t)\right\rangle_{v o l}= & (\alpha+\beta)\left(\frac{4 \gamma \delta}{(\gamma+\delta)^{3}}(\exp [-(\gamma+\delta) t]-1)\right. \\
+ & \left.\frac{2 \gamma \delta}{(\gamma+\delta)^{2}} t \exp [-(\gamma+\delta) t]+\frac{2 \gamma \delta}{(\gamma+\delta)^{2}} t\right)
\end{aligned}
$$

From Eqs. (26) , (27) and (28) we can make the following observations. Firstly, the variance can be decomposed into two contributions, one corresponding to the particle movement 
across the bulk and the other to the surface movement. The dependence of the variance on the time rates parallel to the surface is linear, while in these same relations, the adsorption and desorption rates enter in a more complicated way. The functional form obtained is similar for both movements (which however are nonindependent).

For a large evolution time, the mean square distance or dispersion grows linear with time; each diffusive process has its own slope or growing rate (these slopes are associated with the diffusion coefficient). However, this is an expected behavior due to the model we are using. The other contributions are transient ones, that decay with a time constant $\tau=(\gamma+\delta)^{-1}$, hence, the stronger the adsorption or desorption, the faster is the decay of these contributions.

\section{RESULTS}

In this section we show the theoretical results, including some special numerical procedures, and make the comparison with Monte Carlo simulations. In all simulations we have fixed the following parameters: $\alpha=\beta=\gamma=1$ and $\alpha^{1}=\beta^{1}=0$, and have averaged over $10^{6}$ realizations.

In Fig. 11 we present the theoretical-numerical (that is using a computer program to calculate the inverse Laplace transform [32]), theoretical results obtained from Eq. (24) and simulation results for the temporal evolution of the probability to find the system on the surface for the bilayer case. Here we show the curves for two values of the desorption rate $\delta$. As is apparent from the figure, there is excellent agreement between the theoretical and simulation results. Such excellent agreement indicates that the numerical procedure for the obtention of the inverse Laplace transform is a reliable tool, and that we can trust their results in those cases where analytical results are not accessible (for instance the cases with larger number of layers that we will consider in the following). In Fig. 2, and again for the bilayer case, we depict for the variance $\left(\left\langle r^{2}(t)\right\rangle\right)$, both theoretical and numerical results. Again the agreement is excellent.

In Figs. 3 and 4 we present the $P\left(m, n, l=1 ; t \mid 0,0, l_{0}=1 ; t=0\right)$ and the $\left\langle r^{2}(t)\right\rangle$ but now for a number of layers larger than two. Here we compare the theoretical and numerical results again. We remark that the theoretical results were obtained fitting the inverse Laplace transformation of Eqs. (15) and (??) numerically. Again we have found an 


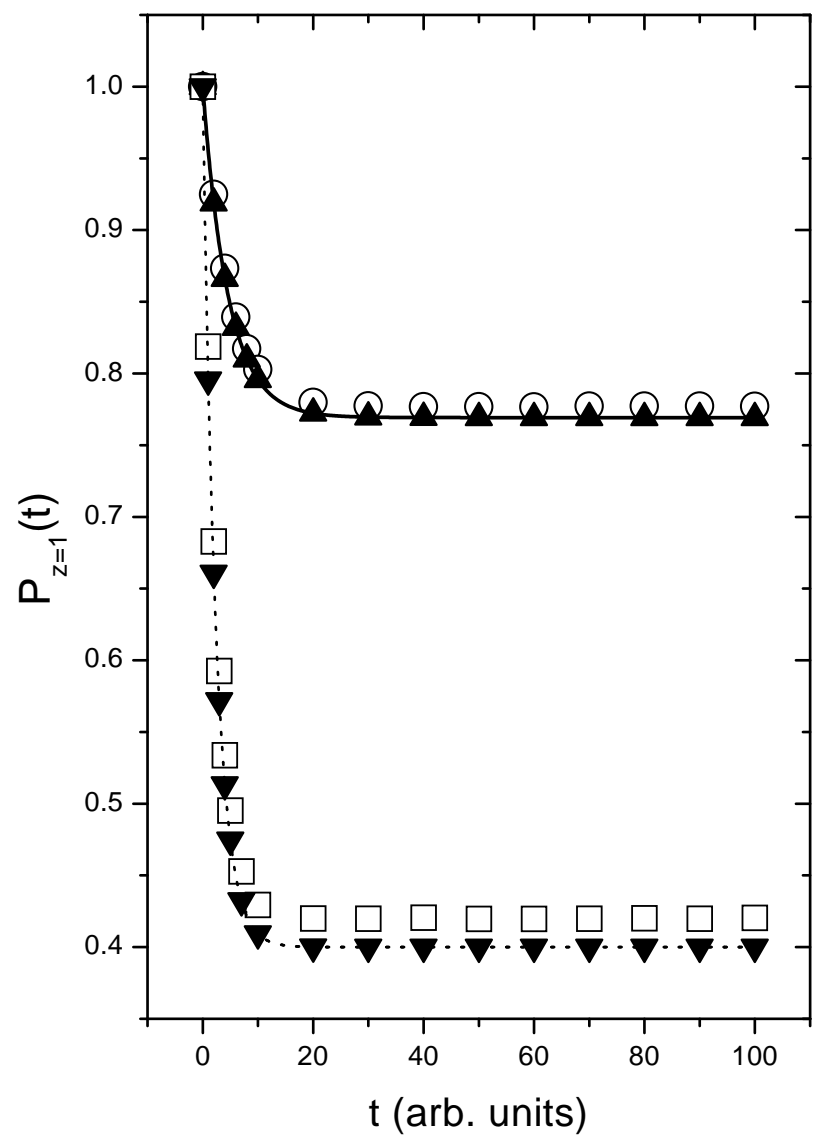

FIG. 1: Temporal evolution of the $P_{z=1}(t)$. We have shown two cases: (i) $\boldsymbol{\Delta}$ represent theoretical points (see Eq. (24)), the continuous line indicates the theoretical-numerical results and $\bigcirc$ are the simulations data for $\delta=0.1$; (ii) $\boldsymbol{\nabla}$ corresponds to theoretical points, the dashed line represents theoretical-numerical results and $\square$ the simulations data for $\delta=0.5$.

excellent agreement between theoretical and simulation results.

Figures 5 , 6 and 7 correspond to an analysis for the asymptotic behavior of the finite system. Figure 5 depicts the curve obtained for the $\left\langle r^{2}\right\rangle$ as a function of $\kappa$ (the number of layers) for three different and large observational times: $t=1200,1300,1500$. The insert shows that for these times, the system is well inside the asymptotic region. As can be seen from the figure, there is a maximum in the motion of the system. In other words, there is an "optimal" number of layers for which the system can spread more rapidly. For a larger 


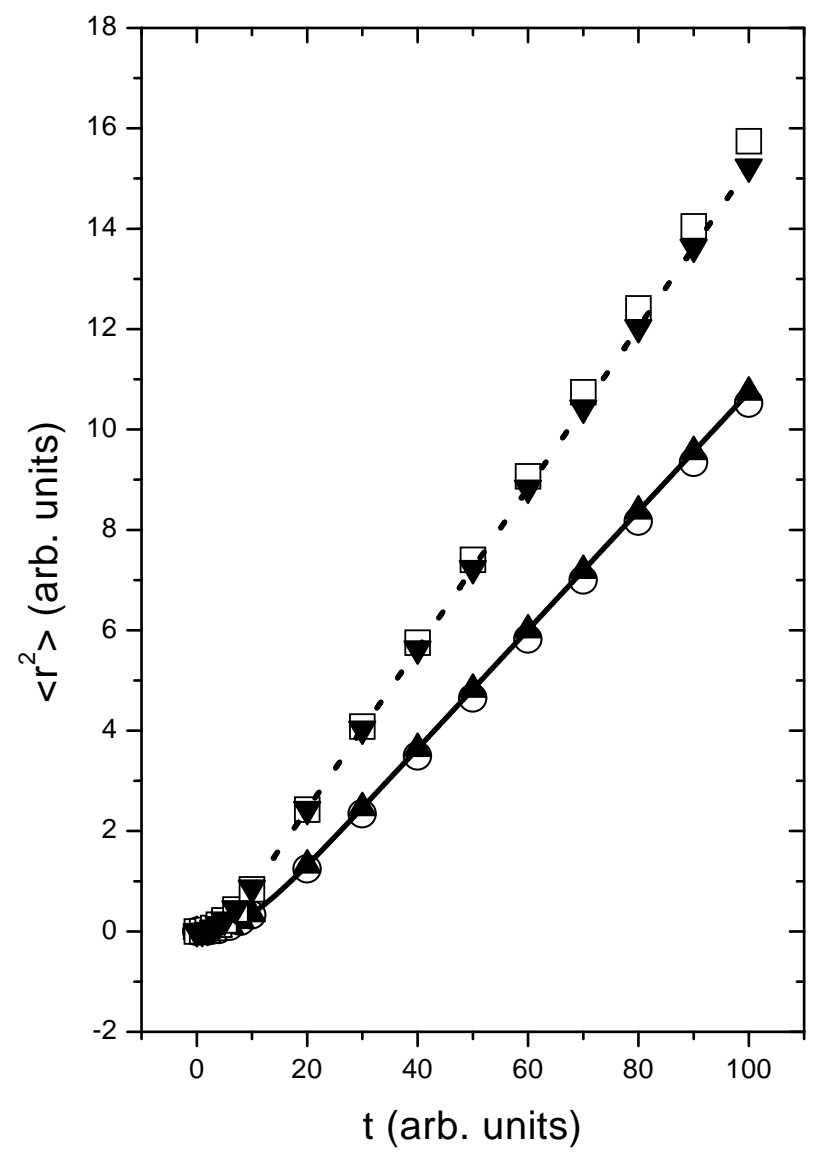

FIG. 2: Time evolution of $\left\langle r^{2}\right\rangle$. We have represented two cases: (i) $\boldsymbol{\Delta}$ represent theoretical points (see Eq.(24)), the continuous line indicates the theoretical-numerical results and $\bigcirc$ are the simulation data for $\delta=0.1$; (ii) $\boldsymbol{\nabla}$ correspond to theoretical points, the dashed line represents theoreticalnumerical results and $\square$ the simulations data for $\delta=0.5$.

number of layers, the system converges to an asymptotic limit. This is reasonable due to the fact that the finiteness of the system tends to disappear.

Figure 6] depicts the same behavior as Fig. [5] but now we have fixed an observational time $(t=1500)$ and we have used the desorption rate $(\delta)$ as parameter. The figure also shows the maximum on the number of layers again but from this figure we can see that this maximum moves towards the lower layers as the desorption rate increases. The insert shows the time evolution of $\left\langle r^{2}\right\rangle$ for large values of $\kappa$. We compare this behavior with the one corresponding 


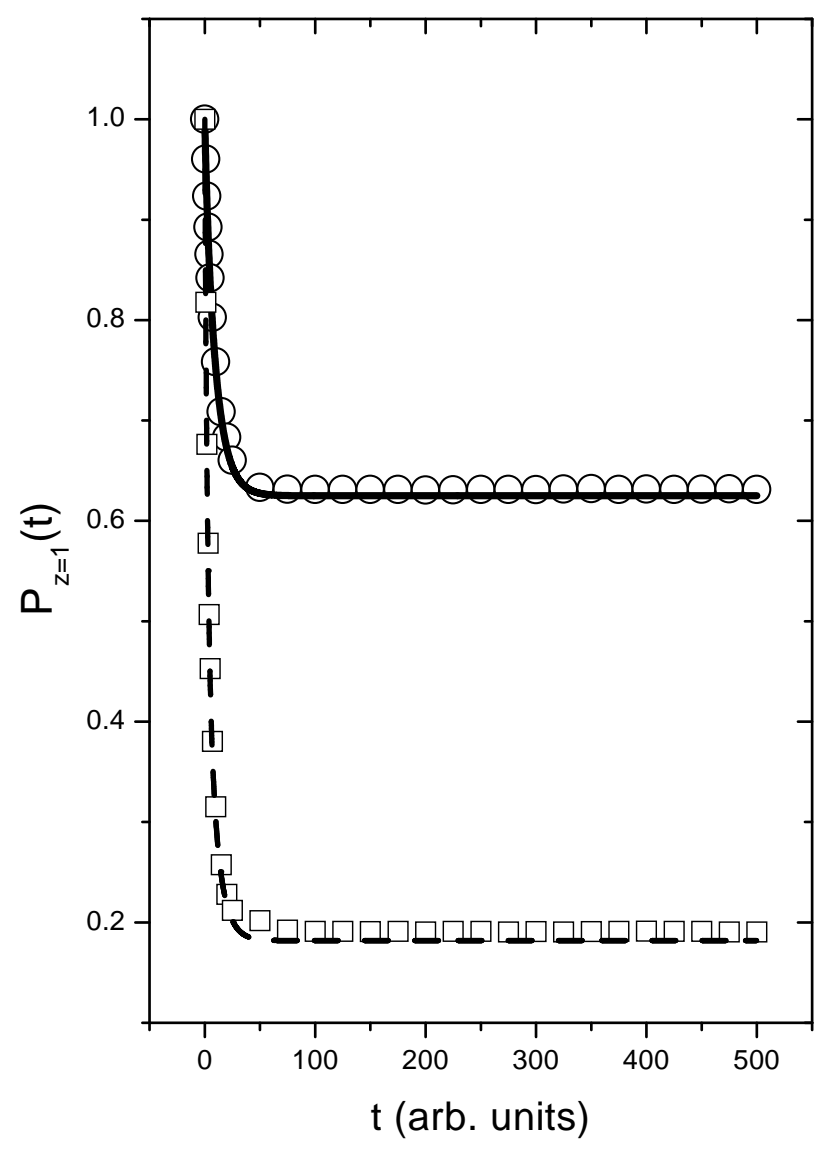

FIG. 3: Time evolution of the $P_{z=1}(t)$. We have represented two cases: (i) the continuous line depicts the theoretical result obtained numerically and $\bigcirc$ are the simulation points for 3 layers; (ii) the dashed line represents theoretical results and $\square$ the simulations for 4 layers.

to the infinite case [27]. From this figure we can say that the finite system behaves, for the used parameters, as an infinite one when the number of layers is $\kappa>50$.

If the parameter $\delta$ is increased, the maximum finally disappears as can be seen in Fig. 7 , This figure shows two curves for two different $\delta$ for $t=1500$. The insert shows the $P_{z=1}(t)$ for these desorption rates.

Finally we show a fitting of $\left\langle r^{2}(t)\right\rangle$ as a function of $\kappa$ for a large evolution time. For 


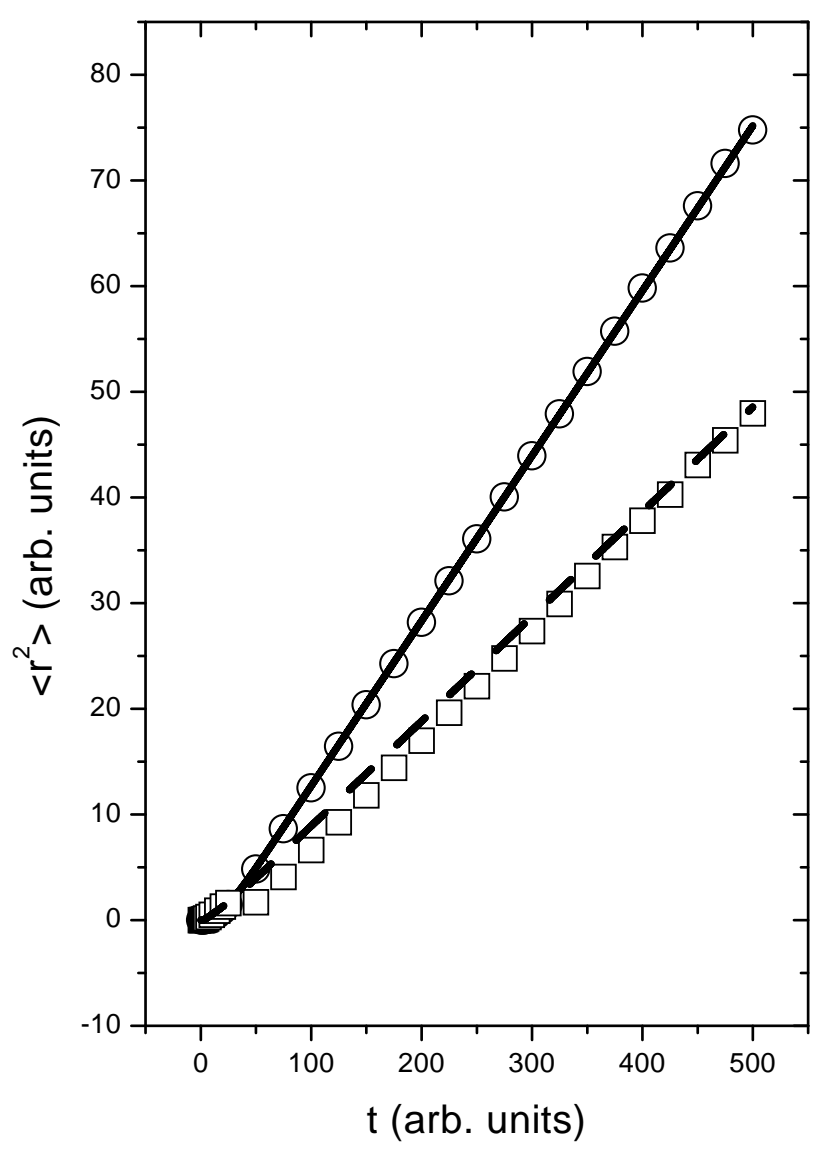

FIG. 4: Time evolution of the variance $\left\langle r^{2}\right\rangle$. We have represented two cases: (i) the continuous line depicts the theoretical result obtained numerically and $\bigcirc$ are the simulation points for 3 layers; (ii) the dashed line represents theoretical results and $\square$ the simulation for 4 layers.

fitting purposes we have used the following function

$$
\left\langle r^{2}(t)\right\rangle=C t^{\epsilon}
$$

where $C$ is a constant and $\epsilon$ is the fitting parameter. In Fig. 8 we depict the results obtained for $\epsilon$ as a function of $\kappa$.

We observe two regions: for $\kappa \leq 20$ the particles describe a diffusion movement. In particular this behavior was shown for the bilayer model. The insert of the figure shows a zoom for a small number of layers, and for two different times. The linear behavior is 


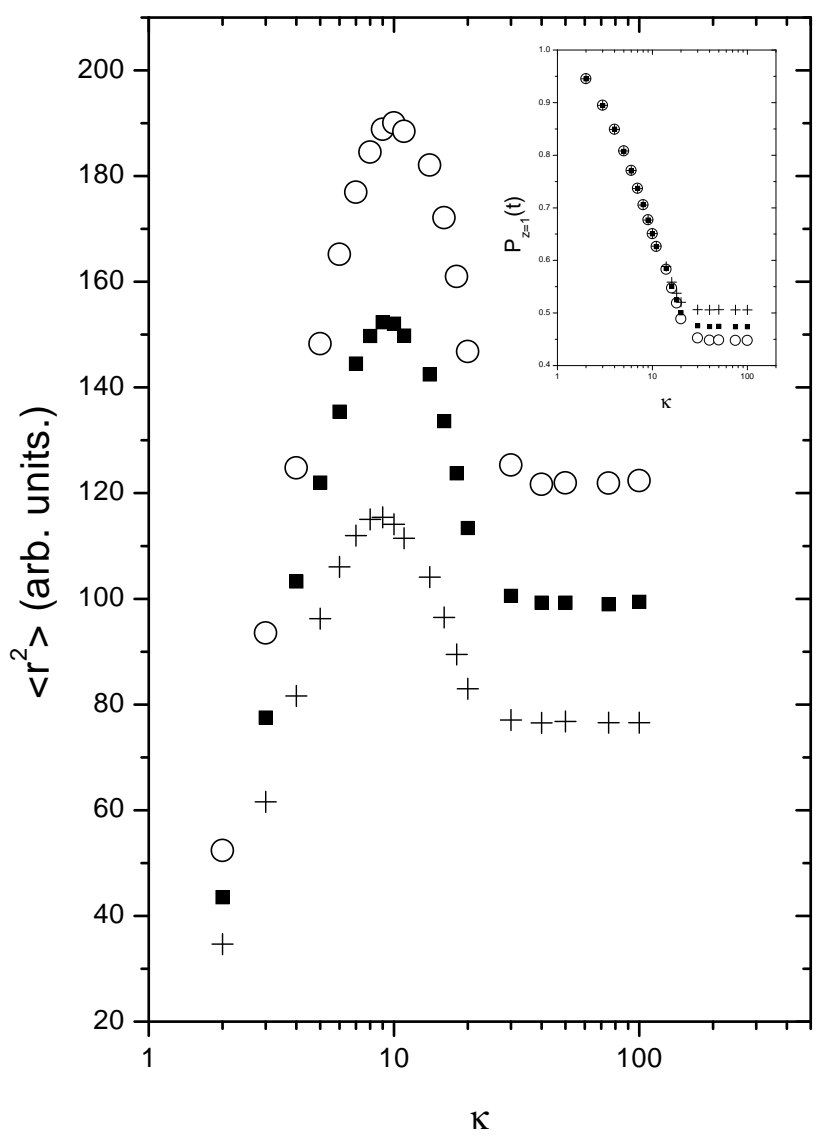

FIG. 5: $\left\langle r^{2}\right\rangle$ vs $\kappa$ for the case $\delta=0.02$. The $\bigcirc$ correspond to an observation time $t=1200, \boldsymbol{\square}$ is for $t=1300$ and + for $t=1500$. The insert shows the $P_{z=1}(t)$ vs $\kappa$. The system is in the asymptotic region.

apparent. A second region appears for $\kappa>20$ where the movement results subdiffusive. For large $\kappa$ the $\epsilon$ parameter approaches an asymptotic value equal to 0.5 . This situation was predicted [27] for infinite systems.

\section{CONCLUSIONS}

We have studied the evolution of particles diffusing in a volume, but have analyzed the statistical properties of their evolution on a surface. The diffusion can be performed on 


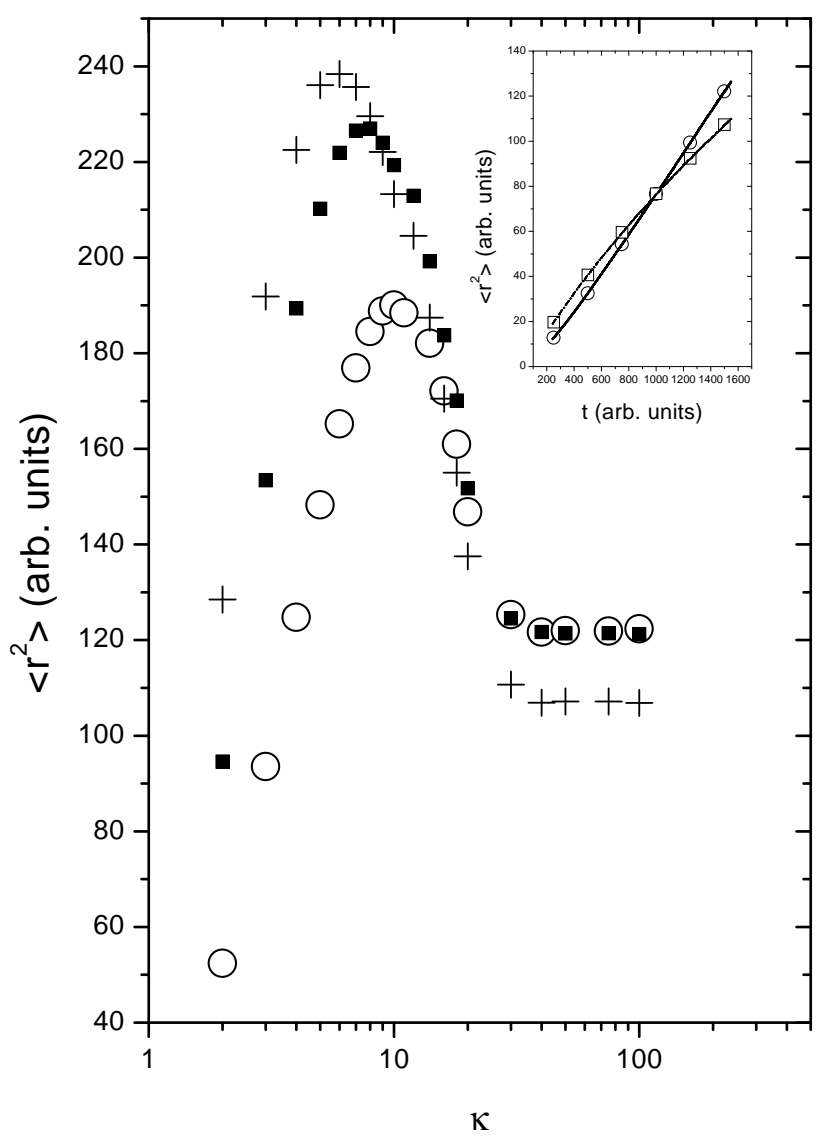

FIG. 6: $\left\langle r^{2}\right\rangle$ vs $\kappa$. We have represented the case for $t=1500$. The $\bigcirc$ correspond to a desorption rate $\delta=0.02$, while $\square$ is for $\delta=0.04$ and + for $\delta=0.06$. Insert: time evolution of $\left\langle r^{2}\right\rangle$ for $\delta=0.02$ and $\kappa=100(\bigcirc)$, and for $\delta=0.06$ and $\kappa=100(\square)$. The continuous lines correspond to the infinite bulk behavior [27].

the surface itself or across the bulk surrounding the surface. In this work we focus on the particle diffusion due to the movement across the bulk, that is we have considered the bulk mediated surface diffusion of the particles. The main feature of the bulk is its finiteness in one direction (the axial one). The other directions are unbounded. This work complements a previous study 27] in which we have analyzed the particle diffusion in a semi-infinite bulk.

Here we have presented a theoretical model based on a set of Master Equations which describe the movement of particles over a simple cubic lattice. This model is general in 


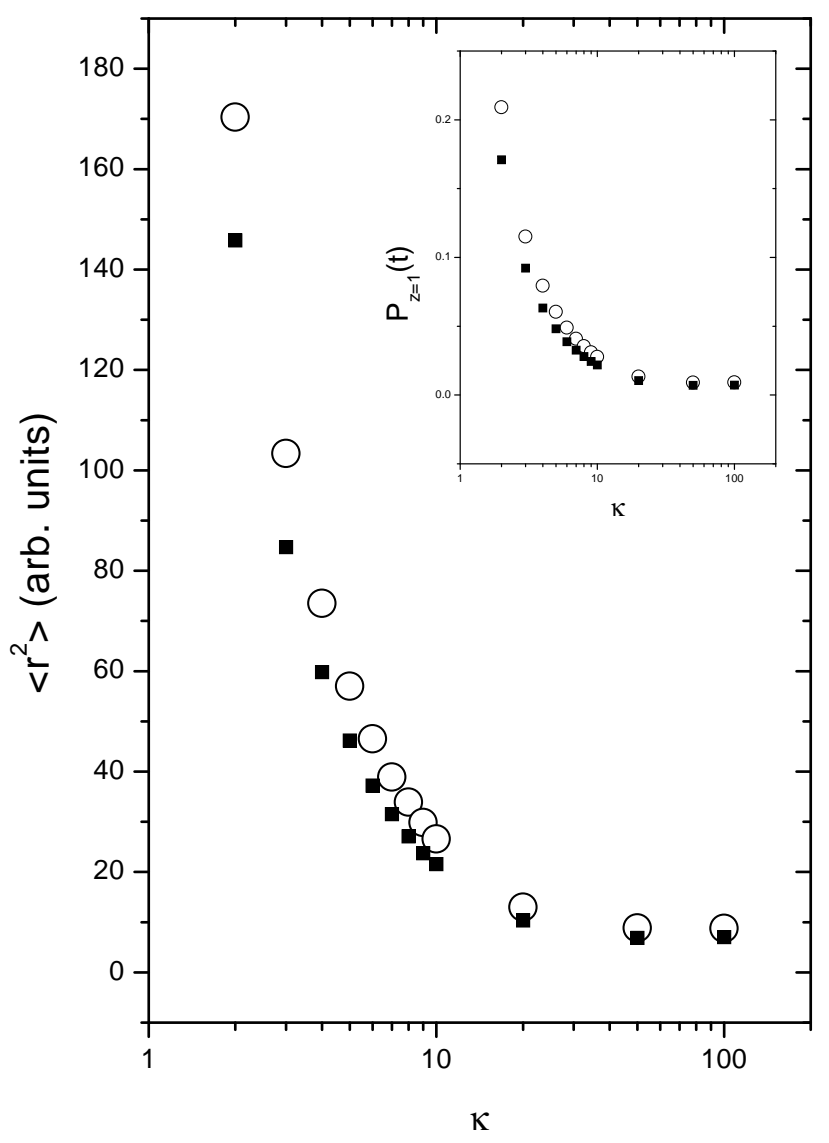

FIG. 7: $\left\langle r^{2}\right\rangle$ vs $\kappa$, The $\bigcirc$ corresponds to a $\delta=1.5$, $\mathbf{\square}$ to a $\delta=2$. Insert: $P_{z=1}(t)$ vs $\kappa$ for these desorption rates.

the sense that we include both kind of particle movement: on the surface and on the bulk. We solved this problem by using techniques of the Laplace and Fourier transformation and exploiting Dyson's formula. It is worth remarking here that the model can describe the evolution of the system everywhere in the bulk, and the evolution of the system for all time. We particularize to the study over a single plane, i.e. the surface. We have obtained general solutions in the Laplace space for the $P_{z=1}(t)$ and the $\left\langle r^{2}(t)\right\rangle$ for any kind of bulk.

In order to find an analytical solution in space and time, we have particularized the problem to the bilayer case. The expressions obtained with this assumption were compared with Monte Carlo simulations, finding excellent agreement. Moreover we were able to handle 


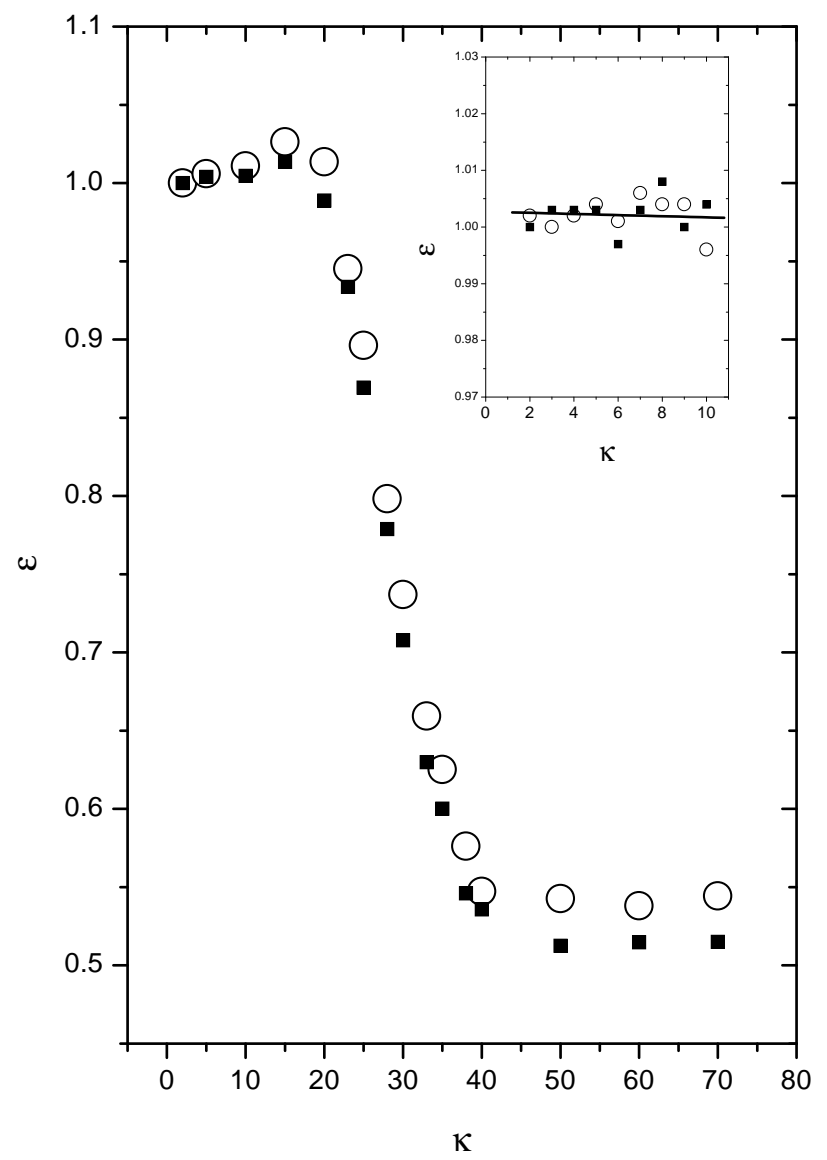

FIG. 8: $\epsilon$ vs $\kappa$. Circles correspond to $\delta=0.5$ and squares to $\delta=2$. The time evolution was $t=2000$. Insert: $\epsilon$ vs $\kappa$ for a small number of layers and $t=2000(\mathbf{a}), t=3000(\bigcirc)$. The line is a linear fitting of the data.

cases with more layers. In these cases, we have obtained the inverse Laplace transform using numerical methods, and compared these results with Monte Carlo simulations. The agreement between both results are excellent again. As indicated above, the numerical procedure to obtain the inverse Laplace transform is a reliable tool, and we have shown that we can trust their results in those cases where analytical results are not accessible.

Finally we have studied the behavior of the spreading in the asymptotic region as a function of the number of layers of the system. We observed an "optimal" number of layers for which $\left\langle r^{2}(t)\right\rangle$ reaches its maximum value. It is worth remarking here that this 
effect occurs in the limit of "strong adsorption", that is when the relation $\frac{\delta}{\gamma}$ is small, and disappears in the limit of "weak adsorption", that is when $\frac{\delta}{\gamma}$ is large.

A possible generalization of the present and the previous related work [27] consists in considering the possibility of non Markovian dynamics, and study the effect of such dynamics on the statistical features of the system. This is the subject of further work.

Acknowledgments: The authors thank V. Grünfeld for a critical reading of the manuscript. HSW acknowledges the partial support from ANPCyT, Argentine, and thanks the MECyD, Spain, for an award within the Sabbatical Program for Visiting Professors, and to the Universitat de les Illes Balears for the kind hospitality extended to him.

[1] V. G. Levich, Physiochemical Hydrodynamics (Prentice-Hall, Inc. Englewood Clliffs NJ. 1962), 2nd ed.

[2] E. H. Lucassen-Reynders and J. Lucassen, Adv. Colloid Interface Sci 2347 (1969).

[3] J. H. Clint Surfactant Aggregation (Chapman and Hall, New Jork, 1992).

[4] D. Languevin, editor, Light Scattering by Liquid Surfaces and Complementary Techniques, (Marcel Dekker, New York, 1992).

[5] E. H. Lucassen-Reynders, J. Lucassen P. R. Garret D. Giles and F. Hollway, Adv. Chem. Scr. 144, $272(1975)$.

[6] C. Stenvot and D. Languevin, Langmuir 41179 (1988).

[7] C. Lemaire and D. Languevin, Colloid Surf. 65101 (1992).

[8] H. E. Johnson, J. F. Douglas and S. Granick, Phys. Rev. Lett. 703267 (1993).

[9] C.T. Shibata and A. M Lenhof, J. Colloid Interface Sci. 148, 469 (1992); ibidem, 148, 485 (1992).

[10] S. Kim and H. Y, J. Phys. Chem. 96, 4034 (1992).

[11] Y. L. Chen, S. Chen, C. Frank and J. Israelachvili, J. Colloid Interface Sci. 153, 244 (1992).

[12] L. Vroman and A. L. Adams, J. Colloid Interface Sci 111, 391 (1986).

[13] J. L. Brash and P. ten Hove, Thromb Haemostasis 51, 326 (1984).

[14] R.J. Rapoza and T.A. Horbett, J. Colloid Interface Sci. 136, 480 (1990).

[15] S. Alexander, J. Phys. France 38, 983 (1977).

[16] P. G. de Gennes, J. Phys. 37, 1445 (1976). 
[17] P. G. de Gennes, Adv. Colloid Interface Sci 27, 189 (1987).

[18] J. F. Douglas, H. E. Johnson and S. Granick, Science 262, 2010 (1993).

[19] B. L. Carvalho, P. Tong, J.S. Huang, T.A. Witten and L. Fetters, J. Macromolecules 26, 4632 (1993).

[20] J.H. Clint, Surfactant Aggregation (Blackie, Glasgow and London, 1992).

[21] L. Netzer and J. Sagiv, J. Am. Chem Soc. 105, 674 (1983).

[22] E.B. Troughton, C.D. Bain and G.M. Whitesides, Langmuir 4, 365 (1987).

[23] R. D. Tilton, C. R. Robertson, and A. P. Gast., J. Colloid Interface Sci. 137, 192 (1990).

[24] L. Kramer J. Chem. Phys. 55, 2097 (1971).

[25] D. Langevin and M. Bouchat, Acad Sci Paris 272, 1422 (1971)

[26] S-Y. Lin, K. McKeigue, and C. Maldarelli, AIChE J. 36 (12), 1785 (1990).

[27] J.A. Revelli, C.E. Budde, D. Prato and H.S. Wio, Bulk Mediated Surface Diffusion: Infinite System Case, Europ. Phys. J. B. (in press).

[28] N.G. van Kampen, Stochastic Processes in Physics and Chemistry, 2nd ed. (North Holland, Amsterdam, 1993).

[29] O. Bychuk, B. O’Shaughnessy, Phys. Rev. Lett. 74, 10, 1795 (1995).

[30] S. Kimmich and R. Seitter, Phys. Rev. Lett. 75, 15, 2855 (1995).

[31] J.A. Revelli, PhD Thesis, Instituto Balseiro, Universidad Nacional de Cuyo, Argentina (2003).

[32] G. Honig and U. Hirdes, On the Application of an Efficient Algorithm for the Numerical Laplace Inversion, Kernforschungsanlage Jülich GmbH, Institut für Festkërperforschung (August 1980). 